\title{
Models including electron correlation in relation to Fock's proposed expansion of the ground-state wave function of He-like atomic ions
}

\author{
M. L. Glasser* \\ Departamento de Física Teórica, Atómica y Óptica, Universidad de Valladolid, ES-47011 Valladolid, Spain and Department of Physics, \\ Clarkson University, Potsdam, New York 13699, USA \\ N. H. March \\ Department of Physics, University of Antwerp, BE-2020 Antwerp, Belgium and Department of Theoretical Chemistry, University of Oxford, \\ Oxford OX1 2JD, United Kingdom
}

L. M. Nieto ${ }^{\dagger}$

Departamento de Física Teórica, Atómica y Óptica, Universidad de Valladolid, ES-47011 Valladolid, Spain (Received 13 December 2010; revised manuscript received 9 November 2011; published 22 December 2011)

\begin{abstract}
Here attention is first drawn to the importance of gaining insight into Fock's early proposal for expanding the ground-state wave function for He-like atomic ions in hyperspherical coordinates. We approach the problem via two solvable models, namely, (i) the $s$-term model put forth by Temkin [Phys. Rev. 126, 130 (1962)] and (ii) the Hookean atom model proposed by Kestner and Sinanoglu [Phys. Rev. 128, 2687 (1962)]. In both cases the local kinetic energy can be obtained explicitly in hyperspherical coordinates. Separation of variables occurs in both model wave functions, though in a different context in the two cases. Finally, a k-space formulation is proposed that should eventually result in distinctive identifying characteristics of Fock's nonanalyticities for He-like atomic ions when both electrons are close to the nucleus.
\end{abstract}

DOI: 10.1103/PhysRevA.84.062119

PACS number(s): 03.65.Ge, 01.55.+b, 02.30.Hq

\section{INTRODUCTION}

Because of the present intractability of the exact analytic solution of the Schrödinger solution for the ground-state of He-like atomic ions, we approach the problem here via two solvable models. The first model we discuss was put forth by Kestner and Sinanoglu [1], who proposed the replacement of the Coulomb confinement form of the external potential $V_{\text {ext }}(\vec{r})$ by a harmonic restoring potential given by $\frac{1}{2} k r^{2}$ but retaining the full Coulombic interaction $e^{2} / r_{12}$. It was natural to refer to this model as the two-electron Hookean atom. The model's great merit is that the center-of mass motion can be separated from the relative motion (see, for instance, Ref. [2]). Kais et al. quote the exact ground-state wave function $\Psi\left(\vec{r}_{1}, \vec{r}_{2}\right)$ for the solvable case of the Hamiltonian $H_{\text {Hooke }}=-\nabla_{1}^{2}-\nabla_{2}^{2}+$ $e^{2} / r_{12}+(k / 2)\left(r_{1}^{2}+r_{2}^{2}\right)$ for $k=\frac{1}{4}$ a.u. as

$$
\Psi\left(\vec{r}_{1}, \vec{r}_{2}\right)=N_{0}\left(1+\frac{1}{2}\left|\vec{r}_{1}-\vec{r}_{2}\right|\right) \exp \left[-\frac{1}{4}\left(r_{1}^{2}+r_{2}^{2}\right)\right],
$$

which corresponds to ground-state energy $E_{0}=$ 2 a.u. $\left(E_{\mathrm{HF}}=2.039325\right.$ a.u., where $E_{\mathrm{HF}}$ represents the Hartree-Fock energy). Following Fock's proposal [3], we immediately rewrite Eq. (1) in hyperspherical coordinates $R$, $\Theta$, and $\alpha$ defined by

$$
\begin{aligned}
& R=\sqrt{r_{1}^{2}+r_{2}^{2}}, \quad r_{1}=R \cos \alpha, \\
& r_{2}=R \sin \alpha, \quad \cos \Theta=\frac{\vec{r}_{1} \cdot \vec{r}_{2}}{r_{1} r_{2}}
\end{aligned}
$$

\footnotetext{
*laryg@ verizon.net

†luismi@metodos.fam.cie.uva.es
}

to obtain

$$
\Psi(R, \alpha, \Theta)=\Psi_{g}(R)-\sqrt{1-\sin 2 \alpha \cos \Theta} \frac{\partial \Psi_{g}(R)}{\partial R},
$$

where $N_{0} \exp \left[-\frac{1}{4} R^{2}\right]=\Psi_{g}(R)$.

For He-like atomic ions Fock proposed expanding the ground-state wave function for small $R$ in a form involving not only non-negative-integer powers of $R$, but also positiveinteger powers of $\ln R$. We refer to the important subsequent work of Refs. [4-9], which have demonstrated the convergence and usefulness of Fock's expansion. It is also relevant here to note two recent papers on the Hookean model [10,11].

Here we note that while Eq. (3) is not directly separable in hyperspherical coordinates, it is the sum of a part $\Psi_{g}(R)$, independent of the angles $\alpha$ and $\Theta$, plus a piece with an $R$ dependence determined by $\partial \Psi_{g}(R) / \partial R$, times the known function $\sqrt{1-\sin 2 \alpha \cos \Theta}$ of angles $\alpha$ and $\Theta$. Clearly, both $R$-dependent parts can be expanded to all orders in $R^{2}$ and there are no logarithmic terms at small $R$. Since for small $R$ both electrons are near the origin of harmonic confinement, it is clear that $e^{2} / r_{12}$ alone for small $r_{12}$ does not lead to Fock-like nonanalytic terms for small $r$. Thus, Coulomb confinement with $V_{\text {ext }}(r)=-Z / r$ replacing the harmonic form needs to be invoked in He if Fock's nonanalytic behavior is to be recovered.

This leads us directly to a second model, proposed by Temkin [12], which is now referred to as the Temkin-Poet [13] model for He-like atomic ions. Particularly relevant to this study is Ref. [14], where it is pointed out that the wave function considered by Howard and March [15], namely,

$$
\Psi_{\mathrm{HM}}\left(\vec{r}_{1}, \vec{r}_{2}\right)=C \exp \left[-(Z-1 / 2)\left(r_{1}+r_{2}\right)+\frac{\left|r_{1}-r_{2}\right|}{2}\right],
$$


is the exact ground-state wave function for the Hamiltonian

$$
\hat{H}_{s}^{\prime}=-\frac{1}{2} \nabla_{1}^{2}-\frac{Z}{r_{1}}-\frac{1}{2} \nabla_{2}^{2}-\frac{Z}{r_{2}}+\frac{1}{r_{>}}+\delta\left(r_{1}-r_{2}\right) .
$$

This differs from the Temkin-Poet model $H_{\mathrm{TP}}=\hat{H}_{s}^{\prime}-\delta\left(r_{1}-\right.$ $r_{2}$ ) by the presence of the $\delta$ function, with the quantity $r_{>}$given by

$$
r_{>}=\frac{1}{2}\left(r_{1}+r_{2}+\left|r_{1}-r_{2}\right|\right)=\max \left(r_{1}, r_{2}\right) .
$$

As put forth in Ref. [12], only the $s$ term in the expansion of $1 / r_{12}$ is retained to obtain the Hamiltonian in Eq. (5). Inserting hyperspherical coordinates from Eq. (2) into the Howard-March (HM) wave function in Eq. (4) then yields

$$
\begin{aligned}
\Psi_{\mathrm{HM}}= & C \exp [-(Z-1 / 2) R(\sin \alpha+\cos \alpha) \\
& \left.+\frac{R}{2}|\sin \alpha-\cos \alpha|\right]
\end{aligned}
$$

with no explicit dependence on $\Theta$.

\section{LOCAL-ENERGY AND KINETIC-ENERGY OPERATORS IN HYPERSPHERICAL COORDINATES}

When the ground-state wave function $\Psi$ is written in terms of the hyperspherical coordinates $R, \alpha$, and $\Theta$ described above, we find it convenient to work with the local $L$ ground-state energy $E_{L}$ defined by

$$
E_{L}=\frac{\hat{H} \Psi(R, \alpha, \Theta)}{\Psi(R, \alpha, \Theta)}=-\frac{\frac{1}{2}\left[\nabla_{1}^{2}+\nabla_{2}^{2}\right] \Psi}{\Psi}+U(R, \alpha, \Theta)
$$

Here $U(R, \alpha, \Theta)$ is the total potential energy entering the Hamiltonian $\hat{H}$. At this point we return to the Hookean atom with $k=1 / 4$ and note immediately that $U_{\text {external }}=U_{\text {ext }}$ is given by

$$
U_{\text {ext }}\left(r_{1}, r_{2}\right)=\frac{1}{8}\left(r_{1}^{2}+r_{2}^{2}\right)=\frac{1}{8} R^{2}
$$

in hyperspherical coordinates. This, of course, is a crucial simplification and means that the entire dependence of $U(R, \alpha, \Theta)$ on $\alpha$ and $\Theta$ for this Hookean atom model comes from the Coulomb repulsion potential energy $e^{2} /\left|\vec{r}_{1}-\vec{r}_{2}\right|$.

Writing the kinetic-energy operator (following, e.g., Macek [16]) in hyperspherical coordinates, the He-atom ground-state Schrödinger equation reads

$$
\begin{aligned}
& -\frac{1}{2}\left[\frac{\partial^{2}}{\partial R^{2}}+\frac{5}{R} \frac{\partial}{\partial R}-\frac{\Lambda^{2}}{R^{2}}\right] \Psi \\
& -\left(\frac{Z e^{2}}{r_{1}}+\frac{Z e^{2}}{r_{2}}-\frac{e^{2}}{r_{12}}\right) \Psi=E \Psi,
\end{aligned}
$$

where $\Lambda^{2}$ is Casimir's operator [cf. Eq. (12)]. We can immediately use the Hookean atom wave function $\Psi$ in Eq. (1) to calculate the kinetic-energy term on the right-hand side of Eq. (8) as

$$
E_{\mathrm{LK}}=\frac{12 \sin ^{2} \Theta+16 \cos \Theta \cos ^{2}(2 \alpha) \csc (2 \alpha)-8 \phi+2\left[r\left(12-R^{2}\right)-12\right] \phi^{2}-\left(R^{4}-16 R^{2}+12\right) \phi^{3}+12 \phi^{4}}{16 R \phi^{3}(\alpha, \Theta)\left[1+\frac{R}{2} \phi(\alpha, \Theta)\right]}
$$

Here $\phi(\alpha, \Theta)=\sqrt{1-\sin (2 \alpha) \cos \Theta}$, while the potentialenergy term $U(R, \alpha, \Theta)$ is

$$
U(R, \alpha, \Theta)=\frac{1}{8} R^{2}+\frac{e^{2}}{R \phi(\alpha, \Theta)} .
$$

Given the explicit kinetic-energy operator

$$
\hat{T}(R, \alpha)=-\frac{1}{2}\left[\partial_{R}^{2}+\frac{5}{R} \partial_{R}+\frac{1}{R^{2}}\left[\partial_{\alpha}^{2}+4 \cot (2 \alpha) \partial_{\alpha}\right]\right],
$$

we write the potential-energy entering $\hat{H}_{s}^{\prime}$ in Eq. (5) as

$$
U(R, \alpha)=-\frac{1}{R}[\mu(\alpha)-\delta(\sin \alpha-\cos \alpha)] .
$$

It should be noted that here we have used the relation

$$
\delta(R x)=\frac{1}{R} \delta(x)
$$

and have defined $\mu(\alpha)$ in Eq. (13) as

$\mu(\alpha)=(Z-1 / 2)(\sec \alpha+\csc \alpha)+\frac{1}{2} \frac{|\sin \alpha-\cos \alpha|}{\sin \alpha \cos \alpha}$.
The total energy is $E_{\mathrm{HM}}=-\left(Z^{2}-Z+1 / 2\right)$, while the local kinetic energy is $E_{\mathrm{HM}}^{K}=\frac{1}{R} \mu(\alpha)-\left(Z^{2}-Z+1 / 2\right)$. The wave function in Eq. (4) has been written in the form

$$
\Psi_{\mathrm{HM}}(R, \alpha)=C e^{-R \lambda(\alpha)},
$$

where

$$
\lambda(\alpha)=(Z-1 / 2)(\sin \alpha+\cos \alpha)-\frac{1}{2}|\sin \alpha-\cos \alpha|
$$

For the HM model it is in $\ln \Psi_{\mathrm{HM}}$ that the variables $R$ and $\alpha$ separate, in contrast to the Hookean atom $\Psi$ in Eq. (3). The quantity $y(\alpha)=\lambda(\alpha)$ must satisfy the two differential equations

$$
\begin{aligned}
y^{\prime \prime} & +4 \cot (2 \alpha) y^{\prime}+5 y \\
& =(2 Z-1)(\sec \alpha+\csc \alpha)+\frac{|\sin \alpha-\cos \alpha|}{\sin \alpha \cos \alpha}
\end{aligned}
$$

and

$$
\left(y^{\prime}\right)^{2}+y^{2}=2\left(Z^{2}-Z+1 / 2\right)
$$




\section{DETAILED TREATMENT OF BOTH MODELS IN k SPACE}

We turn now to the representations of our models in $\mathbf{k}$ space. It is convenient to introduce a dummy integration vector $\vec{R}$ whose magnitude is the coordinate $R$.

The Hookean atom is the simplest to start with. The angle-independent term $\Psi_{g}(R)$ clearly has a Gaussian Fourier transform and the Fourier transform of Eq. (3) is

$$
\begin{aligned}
& \Phi(k, \alpha, \Theta) \\
& =4 \pi N_{0}\left\{2 \sqrt{\pi} e^{-k^{2}}-\left[2+\sqrt{\pi}\left(\frac{1}{k}-2 k\right) e^{-k^{2}} \operatorname{Erfi}(k)\right]\right. \\
& \quad \times \sqrt{1-\sin (2 \alpha) \cos \Theta}\} .
\end{aligned}
$$

[Here $\operatorname{Erfi}(z)$ denotes the error function of an imaginary argument.] Similarly, the Fourier transform of the HowardMarch wave function in Eq. (4) is

$$
\Phi_{\mathrm{HM}}(k, \alpha)=\frac{8 \pi C \lambda}{\left(k^{2}+\lambda^{2}\right)^{2}},
$$

where $\lambda=(Z-1 / 2)(\cos \alpha+\sin \alpha)-\frac{1}{2}|\cos \alpha-\sin \alpha|$.

For the Howard-March and Temkin-Poet models we have

$$
E \Psi(R, \alpha)-U(R, \alpha) \Psi(R, \alpha)=\hat{T}(R, \alpha) \Psi(R, \alpha),
$$

where we write

$$
\begin{gathered}
\hat{T}(R, \alpha)=\frac{1}{2}\left[\Delta_{R}+\frac{1}{R^{2}} \Delta_{\alpha}\right], \quad \Delta_{R}=\partial_{R}^{2}+\frac{5}{R} \partial_{R}, \\
\Delta_{\alpha}=\partial_{\alpha}^{2}+4 \cot (2 \alpha) \partial_{\alpha}, \quad U(R, \alpha)=-\frac{1}{R} f(\alpha),
\end{gathered}
$$

where $f=\mu$ for the Temkin-Poet case and $\mu-2^{-1 / 2} \delta(\alpha-$ $\pi / 4)$ for the Howard-March case. The Fourier transform on the left-hand side of Eq. (20) with respect to the vector $\vec{R}$ gives

$$
E \Phi(k, \alpha)+\frac{f(\alpha)}{2 \pi^{2}} \int \frac{d \overrightarrow{k^{\prime}}}{\left|\vec{k}-\vec{k}^{\prime}\right|^{2}} \Phi\left(\overrightarrow{k^{\prime}}, \alpha\right),
$$

where $\Phi$ denotes the Fourier transform of $\Psi$. We next evaluate the right-hand side of Eq. (20), which can be written, by expressing $\Psi$ as an inverse Fourier transform and expanding the kernel $e^{-i \vec{k} \cdot \vec{R}}$ in spherical Bessel functions,

$$
\begin{aligned}
4 \pi & \int_{0}^{\infty} \hat{T}(R, \alpha)\left[j_{0}\left(k^{\prime} R\right) \Phi\left(k^{\prime}, \alpha\right)\right] k^{\prime 2} d k^{\prime} \\
= & 2 \pi \int_{0}^{\infty}\left\{\left[j_{0}\left(k^{\prime} R\right)+\frac{3}{k^{\prime} R} j_{1}\left(k^{\prime} R\right)\right] \Phi\left(k^{\prime}, \alpha\right)\right. \\
& \left.-\frac{j_{0}\left(k^{\prime} R\right)}{\left(k^{\prime} R\right)^{2}} \Delta_{\alpha} \Phi\left(k^{\prime}, \alpha\right)\right\} k^{\prime 4} d k^{\prime} .
\end{aligned}
$$

The Fourier transform of Eq. (24) is

$$
\begin{aligned}
& \frac{4 \pi^{3}}{k^{2}} \Phi(k, \alpha) \\
& +\frac{12 \pi^{2}}{k} \int_{0}^{\infty}\left\{\left[\ln \left|\frac{k+k^{\prime}}{k-k^{\prime}}\right|-\frac{2 k k^{\prime}}{\left|k^{2}-k^{\prime 2}\right|}\right] \Phi\left(k^{\prime}, \alpha\right)\right. \\
& \left.\quad-\pi k k^{\prime} k_{<} \Delta_{\alpha} \Phi\left(k^{\prime}, \alpha\right)\right\} k^{\prime} d k^{\prime} .
\end{aligned}
$$

Consequently, both the Howard-March and Temkin-Poet model equations have the form

$$
\begin{aligned}
& \left(E-4 \pi^{3} k^{2}\right) \Phi(k, \alpha) \\
& +\frac{f(\alpha)-12 \pi^{3}}{\pi k} \int_{0}^{\infty} \ln \left|\frac{k+k^{\prime}}{k-k^{\prime}}\right| \Phi\left(k^{\prime}, \alpha\right) k^{\prime} d k^{\prime} \\
& +12 \pi^{3} \int_{0}^{\infty} k^{\prime 2} k_{<} \Delta_{\alpha} \Phi\left(k^{\prime}, \alpha\right) d k^{\prime}=0 .
\end{aligned}
$$

Remarkably, Eq. (26) with the Howard-March value of $E$ is a solution of Eq. (20), whereas it is not, for any values of $E$ and $\lambda(\alpha)$, for the Temkin-Poet model. The reason, of course, is that the second derivative in $\Delta_{\alpha}$ operating on Eq. (16) produces the $\delta$ function necessary to cancel that in $f(\alpha)$, but the latter is absent in the Temkin-Poet case.

We have focused on the two model wave functions in Eqs. (3) and (7) in hyperspherical coordinates so far because Eq. (3) for the Hookean atom involves $e^{2} / r_{12}$ exactly, while Eq. (4) has Coulomb confinement through the Hamiltonian $\hat{H}_{s}^{\prime}$ in Eq. (5), but a modified electron-electron interaction in which the $s$-wave term only, namely, $e^{2} / r_{>}$, is retained, plus an additional radial correlation through the $\delta$-function contribution to $\hat{H}_{s}^{\prime}$. The Hookean atom results presented here then make it clear that for small $R$, Fock-like terms in the He atom wave function must arise from an interplay between Coulomb confinement and the full electron-electron interaction $e^{2} / r_{12}$; while Coulomb confinement is exactly incorporated in the model Hamiltonian $\hat{H}_{s}^{\prime}$ in Eq. (5), the electron-electron interaction terms are too primitive to yield terms like $R^{2} \ln R$, for instance, at small $R$.

\section{A THIRD MODEL WITH HARMONIC CONFINEMENT BUT INVERSE SQUARE INTERPARTICLE INTERACTION}

We think it will be of interest to mention here yet a third exactly solvable model in which the wave function itself has clear nonanalytic behavior at small $R$. This is the case for the wave function derived by Crandall et al. [17] for the two-electron model, $H_{c}=-\left(\nabla_{1} 2+\nabla_{2}^{2}\right) / 2+$ $(\omega / 2)\left(r_{1}^{2}+r_{2}^{2}\right)+\lambda / r_{12}^{2}$, which parallels the Hookean atom by having harmonic confinement, but with an inverse quadratic interaction instead of $e^{2} / r_{12}$ as in the Hookean model. The Crandall ground-state wave function $\Psi\left(\vec{r}_{1}, \vec{r}_{2}\right)$ has the explicit form

$$
\Psi_{C}\left(\vec{r}_{1}, \vec{r}_{2}\right)=\exp \left[-m \omega r_{1}^{2} / 2 \hbar\right] \exp \left[-m \omega r_{2}^{2} / 2 \hbar\right] r_{12}^{\gamma},
$$

where $\gamma$ measures the strength of the repulsive coupling through

$$
\gamma=\left[\left(1+4 \lambda m / \hbar^{2}\right)^{1 / 2}-1\right] / 2
$$

For $\gamma$ nonintegral, as is usually the case for this model, we note below two features of the nonanalyticity, reflected in the term $r_{12}^{\gamma}$ (which in hyperspherical coordinates is proportional to $R^{\gamma}$ ), times a known function of $\alpha$ and $\Theta$. First, a physical property, the ground-state density $n(r)=\int \Psi_{C}^{2}\left(\vec{r}, \vec{r}^{\prime}\right) d \vec{r}^{\prime}$, has 
been obtained from Eq. (27) by Capuzzi et al. [18], which for the artificial two-electron atom reads

$n(r)=\frac{(m \omega / \hbar)^{3 / 2}}{2^{a-1} \pi^{3 / 2}} \exp \left[-2 m \omega r^{2} / \hbar\right]_{1} F_{1}\left(\frac{3}{2}+\gamma ; \frac{3}{2} ; \frac{m \omega r^{2}}{\hbar}\right)$.

The corresponding x-ray scattering factor $f(k)$, which is the Fourier transform of $n(r)$, can be calculated analytically from Eq. (29), with the result

$$
f(k)=2 e^{-k^{2} a^{2} / 2}{ }_{1} F_{1}\left(-\gamma ; \frac{3}{2} ; \frac{1}{4} k^{2} a^{2}\right),
$$

where $a=\sqrt{\hbar / 2 m \omega}$. Writing Eq. (27) in hyperspherical coordinates and then taking the Fourier transform with respect to $R$, we find that in $\mathbf{k}$ space, the wave function of Crandall et al. is nonanalytic at small $R$ and has the large-k behavior

$$
\Phi_{C}(k, \alpha, \Theta)=f(\alpha, \Theta) \frac{1}{k^{\gamma+3}},
$$

where $f(\alpha, \Theta)$ is known.

\section{DISCUSSION}

Finally, returning to the specific Fock proposal of $\ln R$ behavior in the He atom, we note that White and Stillinger [19], using first-order perturbation theory on the angle-averaged wave function $\exp \left[-Z^{*} R\right]$ in Ref. [19] as the unperturbed result, found a term $R^{2} \ln R$ times an angular function in He. In this regard, we consider the asymptotic behavior of the Fourier transforms below that are relevant to this work:

$$
\begin{aligned}
F(k)= & \int d R e^{i \mathbf{k} \cdot \mathbf{R}} R^{\alpha} e^{-a R^{2}} \\
= & \frac{2 \pi}{a^{(\alpha+3) / 2}} \Gamma(1+\alpha / 2)_{1} F_{1}\left(\frac{\alpha+3}{2} ; \frac{3}{2} ;-\frac{k^{2}}{4 a}\right) \\
& -\frac{\pi^{3 / 2} 2^{\alpha / 2+2} \alpha}{k^{\alpha+3}} \frac{\Gamma(1+\alpha / 2)}{\Gamma(1-\alpha / 2)},
\end{aligned}
$$

for large $k$ and $\alpha \neq 0,2,4, \ldots$, and

$$
\begin{aligned}
G(k)= & \int d \vec{R} e^{\mathbf{k} \cdot \mathbf{R}} R^{2} \ln R e^{-a R^{2}} \\
= & \frac{\pi \Gamma(5 / 2)}{a^{5 / 2}}\left\{\left.\frac{\partial}{\partial s}{ }_{1} F_{1}\left(s ; \frac{3}{2} ;-\frac{k^{2}}{4 a}\right)\right|_{s=5 / 2}\right. \\
& \left.+\left[\psi \frac{5}{2}-\ln a\right]\left(1-\frac{k^{2}}{6 a}\right) e^{-k^{2} / 4 a}\right\} .
\end{aligned}
$$

For large $k$,

$$
G(k) \sim \frac{2 \pi^{3 / 2}[\ln a-\psi(5 / 2)]}{a^{2}} \frac{e^{-k^{2} / 4 a}}{k} .
$$

The nonanalyticity in Eq. (31) is clearly reflected at large $k$ in Eq. (32), while Eq. (33) is relevant to He itself in the context of the White-Stillinger first-order perturbation calculation [19].

\section{CONCLUSION}

Following Fock's proposal [3], we have expressed the exact ground-state wave function for two solvable models in hyperspherical coordinates. While both wave functions are compact, we have been able to pull them together in $\vec{k}$ space, leading to a common equation [Eq. (26)]. This is one of our principal results. Returning to Fock's ideas though, the Hookean atom model as presented above reveals that in hyperspherical coordinates, Fock-like terms in the He atom wave function for small $R$ can arise only from the interplay between Coulomb confinement and the full electron-electron interaction $e^{2} / r_{12}$. We have also stressed that, while Coulomb confinement is exactly incorporated in the Hamiltonian $\hat{H}_{s}^{\prime}$ in Eq. (5), the electron-electron interaction terms included there are still too primitive to yield terms having, for example, the Fock term $R^{2} \ln R$ at small $R$.

\section{ACKNOWLEDGMENTS}

M.L.G. thanks the Departamento de Física Teórica, Atómica y Óptica, Universidad de Valladolid. N.M.H. acknowledges the University of Valladolid and wishes to thank Professor J. A. Alonso. This work has been supported by the Spanish Ministerio de Educación y Ciencia (Project MTM2005-09183).
[1] N. R. Kestner and O. Sinanoglu, Phys. Rev. 128, 2687 (1962).

[2] S. Kais et al., J. Chem. Phys. 99, 417 (1993).

[3] V. A. Fock, Izv. Akad. Nauk. Arm. SSR, Ser. Fiz. Mat. 18, 161 (1954) [K. Nor. Vidensk. Selsk. Forh. 31, 38 (1958)].

[4] J. H. Macek, Phys. Rev. 160, 170 (1967).

[5] J. Leray, in Trends and Applications of Pure Mathematics to Mechanics, edited by P. G. Ciarlet and M. Roseau, Lecture Notes in Physics Vol. 195 (Springer-Verlag, New York, 1984), p. 235.

[6] J. D. Morgan III, Theor. Chim. Acta 69, 181 (1986).

[7] J. E. Gottschalk and E. N. Maslen, J. Phys. A 20, 2781 (1987).

[8] J. D. Baker, D. E. Freund, R. N. Hill, and J. D. Morgan III, Phys. Rev. A 41, 1247 (1990).

[9] C. R. Myers, C. J. Umrigar, J. P. Sethna, and J. D. Morgan III, Phys. Rev. A 44, 5537 (1991).
[10] P. M. W. Gill and D. P. O’Neill, J. Chem. Phys. 122, 094110 (2005).

[11] P.-F. Loos, Phys. Rev. A 81, 032510 (2010).

[12] A. Temkin, Phys. Rev. 126, 130 (1962).

[13] R. Poet, J. Phys. B 13, 2995 (1980).

[14] C. Amovilli, I. A. Howard, and N. H. March, Phys. Chem. Liq. 46, 238 (2008).

[15] I. A. Howard and N. H. March, Phys. Rev. A 71, 042501 (2005).

[16] J. Macek, J. Phys. B 1, 831 (1968).

[17] R. Crandall, R. Whitnell, and R. Bettega, Am. J. Phys. 52, 438 (1984).

[18] P. Capuzzi, N. H. March, and M. P. Tosi, Phys. Lett. A 339, 207 (2005).

[19] R. J. White and F. J. Stillinger, J. Chem. Phys. 52, 5800 (1970). 